\title{
Rutas de infección por el Virus de Inmunodeficiencia Humana inicialmente no consideradas factibles.
}

Ligia del C. Vera-Gamboa.

Laboratorio de Hematología, Centro de Investigaciones Regionales “Dr. Hideyo Noguchi”, Universidad Autónoma de Yucatán, Mérida, Yucatán, México.

\section{RESUMEN.}

Antecedentes. Desde el inicio de la epidemia del virus de inmunodeficiencia humana fueron identificados los prácticas de riesgo asociadas a la infección, sin embargo, otros mecanismos de transmisión fueron considerados poco factibles como son sexo oral y otros inusuales por ocurrir en situaciones y lugares no ligados a los comportamientos conocidos de riesgo como es la transmisión de persona a persona, transmisión domiciliaria, transmisión nosocomial y de proveedor de salud al cliente entre otros.

Objetivos. Actualizar conceptos a la luz de la revisión de la literatura científica acerca de estos mecanismos dada su importancia en la prevención y control del VIH/Sida y enfatizar la importancia de observar las precauciones universales de prevención.

Resultados. En la actualidad, en la transmisión del VIH a través del sexo oral, es importante considerar los títulos de VIH, la infectabilidad del virus y la integridad de las mucosas. La transmisión nosocomial ha sido reportada y avalada con estudios de biología molecular así como la transmisión de persona a persona. La proporción de accidentes laborales al VIH en México es similar a la de los Estados Unidos. El reporte inicial de 1990 de infección por VIH en un paciente de dentista, ha sido completado con análisis filogenéticos para totalizar seis pacientes. Más recientemente se ha reportado el caso de un cirujano ortopedista infectado en un accidente ocupacional quien infectó a una paciente.

Conclusiones. La práctica del sexo oral es actualmente considerada una práctica de riesgo para la infección por el VIH, lo que cobra relevancia en la actualidad ya que la práctica del sexo oral parece ser una práctica sexual más aceptada por la sociedad que la anal, por lo que se debe enfatizar la promoción y educación del sexo seguro. Hasta el momento no existen evidencias concluyentes de transmisión nosocomial

Solicitud de sobretiros: M.C. Ligia Vera-Gamboa, Depto. de Hematología, Centro de Investigaciones Regionales "Dr. Hideyo Noguchi", Universidad Autónoma de Yucatán, Av. Itzáes \# 490 x 59, C.P. 97000, Mérida, Yucatán, México. E-mail: vgamboa@tunku.uady.mx 


\section{L del C Vera-Gamboa.}

o de persona a persona más que a nivel de epidemiología molecular. Sin embargo, la ocurrencia de los casos presentados avalan la posibilidad de su ocurrencia. Ante este panorama es importante observar, mantener y reforzar las precauciones universales de prevención en el control de la infección de VIH.

(Rev Biomed 2001; 12:122-129)

Palabras clave: VIH, SIDA, sexo oral, transmisión nosocomial del VIH, transmisión ocupacional del VIH.

\section{SUMMARY.}

Human Immunodeficiency Virus infection routes which were not initially considered possible.

Background. Risk factors associated with the human immunodeficiency virus were identified since the beginning of the epidemic. However, other unusual ones, were considered unlikely because they didn't occur in situations or places related to known risk behaviour such as person to person transmission, domiciliary transmission and from health giver to patient among others.

Objectives. To bring concepts about transmission mechanisms up to date for revision by scientific literature given their importance in the prevention and control of HIV/AIDS and to emphasise the importance of observing universal preventatiive precautions.

Results. Nowadays, it is important to consider the titres/titles of HIV, the contagiousness of the virus and the integrity of the mucous linings in the transmission of HIV by oral sex, hospital transmission and person-to-person transmission have been reported and evaluated by molecular biological studies. The proportion of accidental transmission in the workplace in Mexico is similar to that of the United States. The initial report of HIV infection in a dentist's patient in 1990, has been completed by phylogenetic analysis to give a total of six patients. More recently, the cause of an patient arthopaedic surgeon who infected a patient in a occupational accident was reported.

Conclusions. The pracrtice of oral sex is actually considered to be a risk factor for HIV infection, which becomes important in the present since the practice of oral sex seems to be more accepted by society than anal sex therefore the promotion and education of safe sex must be emphasised. So far there is no conclusive evidence of hospital transmission or person-to-person transmission other than at the molecular epidemiological level, however, the occurrence of the presented cases give value to the possibility of their occurrance. In the light of this, it is important to observe, maintain and reinforce the universal preventative precautions in the control of HIV infection.

(Rev Biomed 2001; 12:122-129)

Key words: HIV, AIDS, oral sex, hospital transmission of HIV, accidental transmission of HIV.

\section{INTRODUCCION.}

Desde la aparición de los primeros casos de infección por el virus de inmunodeficiencia humana (VIH), algunos mecanismos de transmisión fueron considerados como no factibles o poco factibles. Entre éstos se encuentran la práctica del sexo oral, la transmisión ocupacional, de persona a persona, la transmisión nosocomial y la transmisión de proveedor de salud al cliente. Se ha acumulado evidencia de que algunos de ellos han pasado de ser considerados de bajo riesgo a ser hoy considerados de riesgo intermedio para la infección del VIH como es el caso de la práctica del sexo oral. Otros mecanismos a pesar de su mínimo riesgo siguen siendo una preocupación para un sector de la población, como es el caso entre otros de la transmisión ocupacional o nosocomial.

\section{TRANSMISION POR SEXO ORAL.}

En un inicio, la información del riesgo real de transmisión a través del sexo oral era

\section{Revista Biomédica}




\section{Rutas de infección del VIH infrecuentes.}

incompleta ya que, en un principio se postuló que la infectabilidad del VIH presente en saliva era baja, mil veces menos que en sangre, y que esto se debía a la presencia de una baja carga viral en comparación con los títulos sanguíneos (1), considerándose una práctica de bajo riesgo. Estudios realizados en el Centro de Control de Enfermedades (CDC) en animales, mostraron que cuando se aplicó concentrados del virus en mucosa rectal, vaginal y encías de monos y chimpancés seronegativos, un chimpancé se había infectado con VIH-1 y un mono con virus de inmunodeficiencia simia a través de mucosa rectal y vaginal, pero no a través de las encías $(2,3)$. Posteriormente, la Dra. P. Folks mezcló VIH en saliva de hombres, mujeres y chimpancés seronegativos en tubos conteniendo linfocitos $\mathrm{T}$, encontrando que en los chimpancés ocurrió un bloqueo completo del VIH, lo que no ocurrió en los humanos, presentándose en éstos sólo un bloqueo parcial de entre 10 a $60 \%$ (1). El motivo de esta variabilidad, está dado por el tipo de glándula que produce la saliva, ya que la producida en las submaxilares y sublinguales inhiben al virus a través de la presencia en altas concentraciones de glicoproteínas, lo que no ocurre con la producida en las glándulas parótidas. Por microscopía electrónica estas moléculas causan aglutinación del VIH disminuyendo de esta manera su infectabilidad. Es importante mencionar que en el cérvix y la vagina no se producen estas sustancias.

Quayle refiere que ha sido reportado aislamiento del VIH en el semen hasta en un 30\%, y un estudio previo realizado por él y su equipo en 95 hombres VIH positivos el virus sólo fue aislado en un 9\%. Posteriormente siguió a 14 hombres VIH positivos quienes se encontraban asintomáticos y fueron vigilados a lo largo de 8 meses, demostrando al final que el VIH pudo ser recuperado en el semen de todos ellos, en algún momento del estudio, postulando que el semen de una persona puede no ser infeccioso en un momento determinado, pero puede serlo en cualquier momento (4).

Otro componente de riesgo orogenital a considerar es el líquido preeyaculatorio. En éste, el VIH ha sido encontrado en el $43 \%$ del preeyaculado de hombres seropositivos (5) detectado a través de pruebas de biología molecular como es la reacción en cadena de polimerasa (PCR), lo que demuestra un riesgo potencial para la infección en la práctica del sexo oral y que ha sido avalado por las siguientes evidencias: existe el reporte de un hombre que adquirió de manera simultánea gonorrea y VIH después de prácticas de fellatio (6). Igualmente, hombres con prácticas de fellatio receptivo (7) y dos confellatio insertivo seroconvirtieron a VIH, así como dos hombres que habían tenido relaciones con hombres $(\mathrm{HSH})$ con prácticas orogenitales, aunque éstos refirieron presencia de eyaculación $(8,9)$.

Respecto a las mujeres es importante considerar que en aproximadamente el 25\% de las mujeres infectadas, están presentes otras infecciones por lo que la suceptibilidad a la infección por el VIH es mayor. Esta suceptibilidad aumenta durante la menstruación, ya que la sangre es altamente infecciosa y el $\mathrm{pH}$ vaginal se modifica con la sangre y el semen (4). Únicamente ha sido reportado en relación al cunnilingus cuatro casos de infección por VIH entre lesbianas (10), en dos de ellos, se reportó que ambas mujeres presentaban sangrado vaginal por actividades sexuales traumáticas $(11,12)$.

Así, es menester considerar los siguientes factores en el riesgo de infección por VIH en prácticas orogenitales: los títulos de VIH, la misma infectabilidad del virus y la integridad de las mucosas. Estas últimas, proporcionan protección a través de defensas tácticas como son la presencia de la flora normal y la secreción de moco espeso, el cual actúa como barrera. Existe un segundo mecanismo como es el sistema inmune mucoso, con la presencia de inmunoglobulina A (Ig A) misma que se encuentra en la sangre sólo en pequeñas cantidades. Esta Ig A además de actuar 


\section{L del C Vera-Gamboa.}

como anticuerpo puede unirse a otras moléculas de Ig A formando así moléculas más complejas lo que puede aumentar su capacidad de neutralización (4). Es importante considerar este hecho ya que recientemente en Tailandia (13) ha sido reportado disrupción de la mucosa vaginal después de la aplicación de ácido metacreosulfónico y formaldehido (Albothyl), pudiendo este hecho facilitar la transmisión del VIH.

Podemos concluir a la luz de estos reportes, que el sexo oral conlleva riesgo importante de transmisión de VIH y ya no puede ser considerada una práctica de bajo riesgo. Además de que el contacto orogenital parece ser una práctica sexual aceptada mas fácilmente por la sociedad, de donde resalta la importancia de la promoción y educación del sexo seguro como ya ha sido reportado previamente $(3,14)$.

\section{TRANSMISION POR SANGRE CONTAMINADA A MUCOSAS.}

Consideraremos ahora la posible transmisión de VIH por exposición de mucosa a sangre contaminada. Es importante señalar que se reporta una prevalencia de $75 \%$ de gingivitis en la población americana (2), la cual probablemente sea mayor entre la población mexicana. Aún cuando como se conoce la saliva pueda bloquear al virus, la presencia de lesiones orales o enfermedades de las encías lesionadas, no siempre obvias podrían ser la ruta directa de entrada del VIH. En 1997 el CDC (15) reportó el caso de una mujer, esposa de un hombre seropositivo. Ella había sido reportada como negativa desde 1988 presentando seroconversión en 1995. Su historial clínico reportó enfermedad periodontal en agosto de 1994 acompañado de cuadro febril y malestar general. Durante el interrogatorio como dato sugestivo de riesgo se registró la práctica de "besos profundos" o muy apasionados, particularmente por la noche después del cepillado dental de su pareja, quien presentaba gingivitis sangrante, presumiéndose que la mezcla de sangre con saliva en una mucosa lesionada fue el mecanismo de transmisión, desde entonces ha sido sugerido no realizar este tipo de prácticas en un período de dos horas posterior al cepillado dental.

Existe otro reporte de un hombre de 36 años quien era seronegativo y fue intervenido por uvuloplastía y tonsilectomía, presentando como complicación sangrado faríngeo en dos ocasiones, tres meses después de la cirugía. El paciente refirió haber reiniciado prontamente su actividad sexual contabilizando 20 parejas casuales, de las cuales dos conocía como seropositivas. Asimismo, refirió prácticas de fellatio receptivo sin condón manifestando haber deglutido líquido preeyaculatorio en una ocasión y negando prácticas orogenitales con eyaculación. En septiembre de 1996 presentó cuadro clínico sin mejoría a pesar de tratamiento, por lo que se le solicitó anticuerpos VIH los cuales se reportaron positivos (16).

\section{TRANSMISION DE PERSONA A PERSONA.}

Entre las diferentes formas de transmisión de VIH, una pelea con sangrado abundante y contacto con piel lesionada también ha sido reportado (17). Esto sucedió después de una pelea violenta y sangrienta entre dos adultos, en la cual hubo intercambio sanguíneo a través de la piel de la frente por rotura de lentes metálicos. De ellos, uno era positivo a VIH y hepatitis B y C. Dos semanas después, el otro protagonista presentó síntomas compatibles con infección primaria por VIH y tres meses después desarrolló hepatitis aguda siendo entonces positivo a la principal proteína estructural del VIH. El análisis filogenético del virus de ambos hombres fueron reportados similares $(18,19)$. Es importante señalar que la epidemiología molecular se considera la herramienta ideal cuando se pretende documentar la transmisión de VIH entre dos personas (20). Diversos estudios han demostrado que las secuencias provirales de la región V3 del VIH no varían mas de un 0 a $5 \%$ entre dos personas involucradas en la transmisión de un 


\section{Rutas de infección del VIH infrecuentes.}

evento en contraste con una diferencia igual o mayor al $10 \%$ si la infección no fue adquirida del paciente fuente $(21,22)$.

\section{EXPOSICION OCUPACIONAL}

Otra preocupación especialmente para el personal del área de la salud, ha sido el riesgo de exposición ocupacional al VIH. El primer caso de seroconversión al VIH, posterior a un accidente ocupacional fue reportado en 1984 (23) y desde entonces hasta finales de 1996 el CDC ha reportado 52 casos documentados de infección y 111 posibles casos por esta vía, los cuales corresponden predominantemente a personal de enfermería y técnicos de laboratorio. En la actualidad se estima el riesgo de transmisión de VIH por exposición percutánea en $0.31 \%$ o de 1 en 325 exposiciones (24).

Estadísticas de la exposición ocupacional al VIH en personal de salud en México, reportaron en 1996 la existencia de ocho casos de Sida por esta vía os por la Secretaría de Salud (SSA). Todos ellos seroconvirtieron posterior al accidente, aunque es de hacer notar que no fueron documentados datos al momento del accidente(25), lo que equivale a una proporción de casos por este mecanismo de $0.03 \%$, cifra similar a la reportada para los Estados Unidos (26). De acuerdo con datos de la SSA, ocurren en nuestro país 33 accidentes por cada 100 camas ocupadas por año, lo que implicaría la posibilidad de ocurrencia de 365661 accidentes ocupacionales anuales, considerando 1'108 064 ingresos a los servicios hospitalarios de esta Secretaría (27).

Robles y col. (Lesiones de riesgo en los trabajadores de la salud. Experiencia del Hospital Angeles. Coordinación de control de infecciones. Trabajo presentado en la 11 Reunión Académica de Aniversario del Hospital) en 1997, reportaron que los accidentes laborales en este país, han disminuido ante la toma de conciencia por parte de los trabajadores de la salud para la observancia de las precauciones universales de prevención. A pesar de ello, es conveniente insistir con cursos de capacitación así como proporcionar las condiciones y materiales necesarios este sector de la población.

\section{TRANSMISION NOSOCOMIAL.}

Asimismo la preocupación se extiende a la posible transmisión nosocomial de VIH de paciente a paciente a través de equipo contaminado. En este renglón se han reportado algunos casos. En 1994 se publicó la infección por VIH en un bebé de término a quien se le practicó circuncisión exatrahospitalariamente. Ingresa posteriormente para tratamiento tópico e intravenoso de conjuntivitis por Clamydia. Es reingresado a los 8 meses por cuadro de insuficiencia respiratoria y pérdida de peso haciéndose el diagnóstico de neumonía por $P$. carinii. La investigación mostró padres y abuelos seronegativos a VIH. En la unidad de aislamiento pediátrico dos de 15 niños ingresados tenían diagnóstico de Sida. En total al bebé se le realizaron 99 procedimientos que involucraron agujas ó contacto con mucosas. En este tiempo, de los niños con Sida a uno se le realizaron 79 procedimientos de este tipo y al otro 6 y a los restantes niños entre 3 a 30 procedimientos similares, por lo que se postula que en algún momento pudo haberse utilizado un equipo contaminado. La causa de infección real permanece desconocida, pero es altamente preocupante esta ruta nosocomial (28).

En Rumania, a principios de la década de los 90, el 85\% de los casos de Sida reportados en este país se registraron en niños menores de 4 años. En el $68 \%$ de estos casos pediátricos la infección ocurrió sin la presencia de factores de riesgo conocidos como transmisión vertical o transfusiones sanguíneas (29). De los más de 2000 casos de Sida registrados hasta los primeros meses de 1993, más de la mitad ocurrió en niños que vivían en orfanatorios. Un estudio realizado por Hersch y col. (30) en niños abandonados, mostró que ante la saturación de trabajo y falta de 


\section{L del C Vera-Gamboa.}

entrenamiento del personal encargado de los niños pudo ocurrir que una misma jeringa pudo ser utilizada para varios niños, posiblemente en la aplicación de viales multidosis de antibióticos. En este estudio se demostró una correlación directa entre el número de inyecciones recibidas y el estado de seropositividad de los niños. En un estudio realizado en 16 niños infectados por VIH después del nacimiento presumiblemente por transmisión nosocomial, la carga viral fue mayor en los niños con diagnóstico de Sida comparada con la de los niños infectados asintomáticos (434 copias vs 68). Es importante recordar que una copia de RNA de VIH-1 puede estar contenida en $0.0013-0.006 \mu \mathrm{L}$ de sangre residual contenida en una aguja (31), dato que podría explicar la posibilidad de transmisión nosocomial.

Más recientemente ha sido reportado en Dinamarca el caso de una niña de 6 años quien contrajo infección por VIH durante su estancia hospitalaria cuando recibía tratamiento para leucemia. En el mismo pabellón, se encontraba un paciente pediátrico con infección por VIH. Las autoridades hospitalarias descartaron una deficiencia en la atención y supusieron la presencia de lesiones durante el juego entre los niños, como arañazos por ejemplo como la causa de transmisión, lo que no pudo ser comprobado. Por técnicas de biología molecular (PCR), se realizó la comparación filogenética de ambos virus, los cuales mostraron una similitud de $97 \%$ (32).

\section{TRANSMISION DE PROVEEDOR DE SALUD A CLIENTE.}

En 1990, el CDC publicó los resultados de una investigación epidemiológica sobre la transmisión de la infección por VIH de un dentista a sus pacientes. Anteriormente este evento no había sido documentado. Este reporte informó de los hallazgos que sugieren infección de VIH en tres pacientes de un dentista con diagnóstico de Sida en Florida (33).

Poco después, otro estudio reportó infección por VIH en tres pacientes más del mismo dentista, totalizando seis pacientes. En estos últimos, al igual que en los tres primeros, no fue posible confirmar datos de exposición a la infección por VIH salvo procedimientos dentales. El análisis filogenético mostró cepas altamente relacionadas entre las de los pacientes y entre los pacientes y el dentista (34). Lo anterior fue confirmado posteriormente en otro estudio apoyando la hipótesis de la transmisión del dentista a paciente (35), sin embargo, a pesar de las evidencias epidemiológicas y virológicas no pudo determinarse fehacientemente en ninguno de estos pacientes la vía de transmisión.

En Francia se reportó el caso de un cirujano ortopedista quien en 1983 sufrió un accidente quirúrgico cuando operaba a un paciente multitransfundido. Más tarde, presentó problemas de salud reportándose seropositivo al VIH, arrojando la investigación epidemiológica el accidente de 1983 como el mecanismo de transmisión. Desde entonces, él había realizado más de 3000 cirugías. De estos pacientes, se reportó una mujer seropositiva sin otros factores de riesgo a excepción de haber sido operada por este cirujano, condición que apoya la posibilidad de transmisión nosocomial. Esta mujer fue intervenida para colocación de prótesis total de cadera con injerto óseo. La cirugía duró alrededor de 10 horas, tiempo mucho mayor al habitual de cualquier otro procedimiento realizado por este cirujano. Al evaluarse las secuencias virales de ambos sujetos éstas fueron muy cercanamente relacionadas (36-38).

\section{CONCLUSIONES.}

Actualmente se conoce que el sexo oral es una práctica de riesgo real para la transmisión de la infección por VIH, mecanismo no considerado al inicio de la epidemia y posteriormente como de bajo riesgo. En la actualidad este tipo de práctica puede ser considerado de bajo a mediano riesgo dependiendo las condiciones en que se realice. Este hecho resalta la importancia de

\section{Revista Biomédica}




\section{Rutas de infección del VIH infrecuentes.}

insistir en la promoción y educación del sexo seguro en la prevención del Sida.

Aunque hasta el momento no existe evidencia concluyente de transmisión nosocomial o de persona a persona más que a nivel de epidemiología molecular, la ocurrencia de los casos expuestos avalan la posibilidad de su presentación particularmente a través de cirugía o procedimientos dentales. De manera ideal en casos de sospecha de transmisión de un paciente a otro debería realizarse pruebas de biología molecular. Finalmente concluir que por estas condiciones de posible transmisión del VIH, es importante mantener y reforzar en todo momento las precauciones universales de prevención.

\section{REFERENCIAS.}

1.- Pinsky L, Douglas P. The essential HIV treatment fact book. Sem Infect Urol 1994; 7:170-83.

2.-Laurence J. The mechanics of transmission. En: The risk of HIV transmission from oral-genital intercourse. Available from: http://www.medscape.com/SCP/IIU/1994/ v07.n06/U438.Lawrence/u438.lawrence.html. Abril 1998.

3.- Waalen J. Oral sex in spotligth for HIV transmission. Ann Intern Med 1996; 125:1-7.

4.- Quayle A. Mucous membrane susceptibility to HIV infection. In: The risk of HIV transmission from oralgenital inetrcourse. Available from: http:// www.medscape.com/SCP/IIU/1994/v07.n06/ U438.Lawrence/u438.lawrence.html. Abril 1998.

5.- Ilaria G, Schlegel P. Detection of HIV-1 DNA in the pre-ejaculate. en: The risk of HIV transmission from oralgenital intercourse. Available from: http:// www.medscape.com/SCP/IIU/1994/v07.n06/ U438.Lawrence/u438.lawrence.html. Abril 1998.

6.- Murray AB, Greenhouse PR, Nelson WL, Norman JE, Jeffries DJ, Anderson J. Coincident acquisition of Neisseria gonorrhoeae and HIV fron fellatio ( letter) Lancet 1991; 338: 830.

7.- Schacker T, Collier A, Hughes J, Shea T, Corey L. Clinical nad epidemiologic features of primary HIV infection. Ann Intern Med 1996; 125: 257-64.
8.- Lifson AR. HIV transmission through specific oralgenital sexual practices. en: The risk of HIV transmission from oral-genital intercourse. Available from: http:// www.medscape.com/SCP/IIU/1994/v07.n06/ U438.Lawrence/u438.lawrence.html. Abril 1998.

9.-Samuel MC, Hessol N, Shiboski S, Engel RR, Speed T, Winkelstein W. Factors associated with human inmunodeficiency virus seroconversion in homosexual men in three San Francisco Cohort Studies, 1984-1989. JAIDS 1993; 6:303-12.

10.- Rich JD, Buck A, Tuomala RE, Kazajian PH. Transmission of human immunodeficiency virus infection presumed to have occurrred via female homosexual contact. Clin Infect Dis 1993; 17:1003-5.

11.-Sabatini MT, Pate K, Hirschaman R. Kaposi's sarcoma and T-cell lymphoma in an inmunodeficiency woman: a case report. AIDS Res 1984;1:135-7.

12.- Marmor M, Weiss LR, Lyden M. Possible femaleto female transmission of human inmunodeficiency virus (letter). Lancet 1985; 2:969.

13.- Kilmarx P, Limpakarnjaranat K, Supawitkul S, Korattana S, Young NL, Parekh BS, et al. Mucosal disruption due to use of a widely-distributed commercial vaginal product: potential to facilite HIV transmission. AIDS 1998; 12:767-73.

14.- Castro-Sansores C. Nuevas evidencias de transmisión por el virus de la inmunodeficiencia humana (VIH). Rev Biomed 1996; 7:187-8.

15.- Center for Disease Control and Prevention. Transmission of HIV possibly associated with exposure of mucous menbrane to contamined blood. MMWR 1997; 46:620-3.

16.- Berrey MM, Shea T. Oral sex and HIV transmission. JAIDS 1997; 14: 475.

17.- O'Farrell N, Tovey SJ, Morgan-Capner P. Transmission of HIV-1 infection after a fight (letter). Lancet 1992; 339:246.

18.- Brambilla A, Pristera R, Salvatori F, Poli G, Vicenzi E. Transmission of HIV-1 and HCV by head-butting (letter). Lancet 1997; 350:1370.

19.- Brambilla A, Salvatori F, Pristera F, Alfano M, Vicenzi E. Molecular analysis of HIV-1 related in a case Vol. 12/No. 2/Abril-Junio, 2001 


\section{L del C Vera-Gamboa.}

of a viral transmission during a bloody fight betwen two adults. AIDS 1998; 12:1928-30.

20.- Fitzgibbon JE, Infrequent patterns of HIV transmission: infection control implications. The AIDS Reader 1995; 5:80-6.

21.- Ou CY, Ciesielski CA, Myers G . Molecular epidemiology of HIV transmission in a dental office. Science 1992; 256:1165-71.

22.-Simmonds P, Balfe P, Ludlam CA, Bishop J, Leigh BA. Analysis of sequence diversity of hypervariable regions of the external glycoprotein of human inmunodeficiency virus type 1. J Virol 1990: 64:5840-50.

23.- Center for Disease Control and Prevention. Recommendations for prevention of HIV transmission in health-care settings. MMWR 1987; 21:451-60.

24.- Larkin JA, Ubillos SS, Sinnott JT, Houston SH. Infection prophylaxis after occupational exposure to HIV: a simple approach. The AIDS Reader 1998; 8:95-8.

25.- Ponce de León S, Del Río ChC, Rangel S, Magis C. Infección por VIH en trabajadores de la salud en México. SIDA/ETS 1996; 2:14-6.

26.- López-Martínez C, Magis-Rodríguez C, HernándezTepichin G. La exposición ocupacional en México. En: Guía de prevención y tratamiento para la exposición ocupacional al VIH. Uribe-Zuñiga P, editor. México: Conasida. 1998; pp. 27-30.

27.- Jagger J. Mecanismos para prevenir las exposiciones ocupacionales a patógenos sanguíneos: observaciones del ambiente laboral de los profesionales de la salud. SIDA/ ETS 1996; 1:82-7.

28.- Blank S, Simmonds RJ, Weisfusse Y, Rudnick J, Chiasseon MA, Thomas P. Possible nosocomial transmission of HIV. Lancet 1994; 344:512-4.

29.- Apetrei C, Buzdugan I, Mitroi I, Ducca M. Nosocomial HIV-1 transmission and primary prevention in Romanian children. Lancet 1994; 340:1028-9.

30.- Hersh BS, Popovici F, Jezek Z, Satten GA, Apetrei RC, Beldescu N, et al. Risk factors for HIV infection among abandoned Romanian children. AIDS 1993; 7:1617-24.
31.- Apetrei C, Descamps D, Panzani C, Ducca MC, Brun-Venizet S, Brun-Venizet F. Plasma HIV-1 load aand nosocomial transmission in Romanian children. AIDS 1995; 9:977.

32.- Skovmand K. HIV-1 infecction puzzles Denmark. Lancet 1997; 350:1758.

33.-Centers for Disease Control and Prevention. Possible transmission of human immunodeficiency virus to a patient during an invasive dental procedure. MMWR 1990; 39:489-93.

34.- Centers for Disease Control and Prevention. Update: Transmission of HIV infection during invasive dental procedures-Florida. MMWR 1991; 40:377-81.

35.-Ciesielski C, Marianos D, Ou Ch, Dumbaugh R, Witte $\mathrm{J}$, Berkelman R, et al. Transmission of human inmunodeficiency virus in a dental practice. Ann Intern Med 1992; 116:798-805.

36.- Holmes EC, Zhang LQ, Simmonds P, Smith RA, Leigh BAJ. Molecular investigation of human immunodeficiency virus (HIV) infection patient of a HIV -infected surgeon. J Infect Dis 1993; 167:1411-4.

37.- Blanchard A, Ferris S, Chamaret S, Guetard D and Montagnier L. Molecular evidence for nosocomial transmission of human inmunodeficiency virus from a surgeon to one of his patient. J Virol 1998; 72:4537-40.

38.- Lot F, Séguier JC, Fégueux S, Astagneu P, Simon P, Aggoune $\mathrm{P}$, et al. Probable Transmission of HIV form an orthopedic surgeon to a patient in France. Ann Intern Med 1999; 130:1-6.

\section{Revista Biomédica}

